九州大学学術情報リポジトリ

Kyushu University Institutional Repository

\title{
A MARKOV DECISION PROCESS WITH NON-STATIONARY TRANSITION LAWS
}

Furukawa, Nagata

https://doi.org/10.5109/13030

出版情報: 統計数理研究. 13 (1/2)，pp.41-52，1968-03. Research Association of Statistical Sciences

バージョン :

権利関係 : 


\title{
A MARKOV DECISION PROCESS WITH NON-STATIONARY TRANSITION LAWS
}

\author{
By
}

\author{
Nagata Furukawa
}

(Received Dec. 15, 1967)

\section{§ 1. Introduction.}

In most of works on Markov decision process ([1], [2], [3] etc.) Markov transition laws of states are assumed to be stationary in the sense that the transition law at any time depends on the state and the action at present time but not on the time. This stationarity of motion law yields some elegant properties on optimal strategies in the discounted case: In [2] Blackwell has proved that in the discounted case there was a $(p, \varepsilon)$-optimal stationary strategy for any initial distribution $p$ and any $\varepsilon<0$, especially in the case of a countable action space there was an $\varepsilon$-optimal stationary strategy for any $\varepsilon>0$, and in the case of a finite action space an optimal stationary strategy. In [3] Strauch has cleared the stationary property of a strong $(p, \varepsilon)$-optimal strategy in the discounted case.

In this paper we shall be concerned with a Markov decision process with nonstationary Markov transition laws, and we shall study the existence and the properties of an optimal strategy, the existence of a strong $(p, \varepsilon)$-optimal strategy, and the strategy improvements.

Our decision problem is based on four objects $S, A, q=\left\{q_{1}, q_{2}, \cdots\right\}$ and $r . S$ and $A$ are non-empty Borel sets, each $q_{j}$ is a conditional probability distribution on $S$ given $S \times A$, and $r$ is a bounded Baire function on $S \times A \times S$. Here $S$ is the set of states, $A$ the set of feasible actions, $q$ the sequence of Markov transition laws of states, and $r$ a reward function. When the system is at a time $j$ and in a state $s$ and an action $a$ is taken, the system moves to a new state according to the conditional probability distribution $q_{j}(\cdot \mid s, a)$, and if the system moves to a new state $s^{\prime}$, then we shall receive a reward $r\left(s, a, s^{\prime}\right)$. In this situation we wish to maximize the total discounted expected reward over the infinite future.

A strategy $\pi$ is a sequence $\pi_{1}, \pi_{2}, \cdots$, where $\pi_{j}$ is a conditional probability distribution on $A$ given $\left(s_{1}, a_{1}, \cdots, a_{j-1}, s_{j}\right)$ for each $j$, and $\pi$ is denoted by $\left\{\pi_{1}, \pi_{2}, \cdots\right\}$. A Markov strategy is a sequence $f_{1}, f_{2}, \cdots$, where each $f_{j}$ is a measurable mapping from $S$ to $A$, and a l-stationary strategy is a Markov strategy such that $\pi=\{\bar{f}, \bar{f}, \bar{f}, \cdots\}$ where $\bar{f}=\left\{f_{1}, f_{2}, \cdots, f_{l}\right\} . \quad q$ is called $l$-stationary if $q=\{\bar{q}, \bar{q}, \bar{q}, \cdots\}$ with $\bar{q}=\left\{q_{1}, q_{2}, \cdots, q_{l}\right\}$.

Our main results are the following: For any initial probability distribution $p$, any $\varepsilon>0$ and any $q$ there is a $(p, \varepsilon, q)$-optimal Markov strategy, i. e. there is a Markov strategy $\pi^{*}$ for which $p\left\{I\left(\pi^{*}, q\right) \geqq I(\pi, q)-\varepsilon\right\}$ for every strategy $\pi$, where $I(\pi, q)$ denotes the total discounted expected reward from a strategy $\pi$ (Section 4 ). If for each $j \geqq 0$ 
there is a $\left(\varepsilon,{ }^{j} q\right)$-optimal strategy, there is a $(\varepsilon /(1-\beta), q)$-optimal Markov strategy, where $\varepsilon \geqq 0$ and ${ }^{j} q=\left\{q_{j+1}, q_{j+2}, \cdots\right\}$ (Section 4). For each $j \geqq 0{ }^{j} \pi^{*}$ is a ${ }^{j} q$-optimal if and only if the total discounted expected reward from $\pi^{*}$ satisfies the system of optimality equations, where ${ }^{j} \pi^{*}=\left\{\pi_{j+1}^{*}, \pi_{j+2}^{*}, \cdots\right\}$ for $\pi^{*}=\left\{\pi_{1}^{*}, \pi_{2}^{*}, \cdots\right\}$ (Section 4). If there exists a ${ }^{j} q$-optimal strategy for each $j \geqq 0$, then there exists a $q$-optimal Markov strategy. If $A$ is essentially countable, there is a $(\varepsilon, q)$-optimal Markov strategy for every $\varepsilon>0$ and every $q$, and if $A$ is essentially finite, then there is a $q$-optimal Markov strategy for every $q$ (Section 4). Eespecially in the case when $q$ is $l$-stationary, all of the results in Section 4 stated above hold by putting a $l$-stationary strategy in place of a Markov strategy (Section 5). Every sequence of Markov strategies is strongly $\varepsilon$-dominated by a Markov strategy for every $\varepsilon>0$ (Section 6 ). For any $p$, $\varepsilon>0$ and any $q$, there is a strong $(\phi, \varepsilon, q)$-optimal Markov strategy (Section 6). In Section 7 there are given several theorems on the improvement of strategies.

In [3] Strauch has derived all of his results on the strong optimality, i. e. the strong $(p, \varepsilon)$-domination, the existence of the strong $(p, \varepsilon)$-optimal Markov strategy and of the strong $(p, \varepsilon)$-optimal stationary strategy etc., with the help of a "conservation". But our proofs of results on the strong optimality are more direct without appealing to the "conservation" (Section 6).

\section{§2. Probabilistic definitions.}

By a Borel set we mean a Borel subset of some complete separable metric space. The class of all probability distributions on $X$ is denoted by $P(X)$. For any nonempty Borel sets $X, Y$ a conditional probability distribution on $Y$ given $X$ is a function $q(\cdot \mid \cdot)$ such that for each $x \in X, q(\cdot \mid x)$ is a probability distribution on $Y$ and for each Borel set $B \subset Y, q(B \mid \cdot)$ is a Baire function on $X$. The class of all conditional probability distributions on $Y$ given $X$ is denoted by $Q(Y \mid X)$. The product space of $X$ and $Y$ will be denoted by $X Y$. The class of bounded Baire functions on $X$ is denoted by $M(X)$. For any $u \in M(X Y)$ and any $q \in Q(Y \mid X)$, qu denotes the element of $M(X)$ whose value at $x_{0} \in X$ is $q u\left(x_{0}\right)=\int u\left(x_{0}, y\right) d q\left(y \mid x_{0}\right)$. For any $p \in P(X)$ and any $u \in M(X)$, $p u$ denotes the integral of $u$ with respect to $p$.

For any $p \in P(X), q \in Q(Y \mid X), p q$ is the probability distribution on $X Y$ such that, for every $u \in M(X Y), p q(u)=p(q u)$. Every probability distribution $m$ on $X Y$ has a factorization $m=p q ; p$ is unique and is just the marginal distribution of the first coordinate variable with respect to $m ; q$ is not quite unique; it is a version of the conditional distributiontion of the second coordinate variable given the first. These facts are given in [4].

We extend the above notation in an obvious way to a finite or countable sequence of non-empty Borel sets $X_{1}, X_{2}, \cdots$. If $q_{n} \in Q\left(X_{n+1} \mid X_{1} \cdots X_{n}\right)$ for $n \geqq 1$ and $p \subseteq P\left(X_{1}\right), p q_{1} q_{2} \cdots q_{n}$ is a probability distribution on $X_{1} X_{2} \cdots X_{n+1}, p q_{1} q_{2} \cdots$ is a probability distribution on the infinite product space $X_{1} X_{2} \cdots, q_{2} q_{3} \in Q\left(X_{3} X_{4} \mid X_{1} X_{2}\right)$, for any $u \in M\left(X_{1} X_{2} \cdots X_{n+1}\right), n \geqq 1$, and any $m, 1 \leqq m \leqq n, q_{m} \cdots q_{n} u \in M\left(X_{1} \cdots X_{m}\right)$, etc.

For the sake of simplicity, we introduce the following ambiguity: for any function $u$ on $Y$, we shall use the same symbol $u$ to denote the function $v$ on $X Y$ such that 
$\imath(x, y)=u(y)$ for all $y$. Thus, for example, for any $q \in Q(Y \mid X), u \subseteq M(Y), q u \in M(X)$; any $q \equiv Q(Y \mid X)$ will also denote the element $q^{\prime}$ of $Q(Y \mid Z X)$ defined by $q^{\prime}(\cdot \mid z, \cdot)$ $=q(\cdot \cdot)$, etc.

A $D \equiv P(X)$ is degenerate if it is concentrated at some one point $x \in X$; a $q \equiv Q(Y \mid X)$ is depenerate if each $q(\cdot \mid x)$ is degenerate. The degenerate $q$ are exactly those for which there is a Baire function $f$ mapping $X$ into $Y$ for which $q(\{f(x)\} \mid x)=1$ for all $x \in X$. Any such $f$ will also denote its associated degenerate $q$, so that, for any $u \in M(X Y), f u(x)=u(x, f(x))$ for ail $x \in X$.

We shall use the following.

LEMMA 2.1 (Blackwell [2]). For any $q=Q(Y \mid X), u \in M(X Y)$, there is a degenerate $f \equiv Q(Y \mid X)$ such that

$$
f u \geqq q u \quad \text { for all } x \in X \text {. }
$$

\section{§3. Decision problem definitions.}

Our dynamic programming problem is defined by $S, A, q, r$ where $S, A$ are any non-empty Borel sets, $q=\left\{q_{1}, q_{2}, q_{3}, \cdots\right\}, q_{i} \in Q(S \mid S A)$ for $i=1,2,3, \cdots, r \in M(S A S)$, and $0<\beta<1$. A strategy $\pi$ is a sequence $\left\{\pi_{1}, \pi_{2}, \pi_{3}, \cdots\right\}$, where $\pi_{n} \in Q\left(A \mid H_{n}\right)$ and $H_{n}=S A S A \cdots S(2 n-1$ factors $)$ is the set of possible histories of the system when the $n$-th act must be chosen. A strategy $\pi$ is Markov if each $\pi_{n}$ is a degenerate element of $Q(A \mid S)$, i. e. $\pi=\left\{f_{1}, f_{2}, f_{3}, \cdots\right\}$, where each $f_{n}$ is a Baire function from $S$ into $A$, and is l-stationary if it is Markov and $\pi=\{\bar{f}, \bar{f}, \cdots\}$ with $\bar{f}=\left\{f_{1}, f_{2}, \cdots, f_{l}\right\}$. The $l$-stationary strategy decided by $\vec{f}$ is denoted by $\vec{f}(x)$.

For any strategy $\pi$, let ${ }^{n} \pi=\left\{\pi_{n+1}, \pi_{n+2}, \cdots\right\}$ denote the strategy which $\pi$ defines from the $(n+1)$-th stage onward. In particular, ${ }^{0} \pi=\pi$. And let ${ }^{n} q=\left\{q_{n+1}, q_{n+2}, \cdots\right\}$.

Any strategy $\pi$, together with the law of motion $q$, defines a conditional probability distribution on the set $X=A S A S \cdots$ of future of the system given the initial states $s$; i. e. it defines

$$
e_{\pi}=\pi_{1} q_{1} \pi_{2} q_{2} \cdots \in Q(X \mid S) .
$$

Any reward function $r$ defines an expected reward function on $S$ gixen by

For any $v \in M(S)$, let

$$
I(\pi, q)=e_{\pi} \sum_{j=1}^{\infty} \beta^{j-1} r\left(s_{j}, a_{j}, s_{j+1}\right) .
$$

$$
I_{n}(\pi, q, v)=e_{\pi}\left[\sum_{j=1}^{n} \beta^{j-1} r\left(s_{j}, a_{j}, s_{j+1}\right)+\beta^{n} v\right] .
$$

We shall denote $I_{n}(\pi, q, 0)$ by $I_{n}(\pi, q)$. Let $Q^{*}$ denote the class of all sequences $\left\{q_{1}, q_{2}, \cdots\right\}$ such that $q_{n} \in Q(S \mid S . A)$ for $n=1,2, \cdots$.

It is clear that

LEMMA 3.1. $I_{n}(\pi, q, v) \rightarrow I(\pi, q)$ as $n \rightarrow \infty$ for any $v \in M(S)$ and any $q \in Q^{*}$.

For any $p \in P(S)$, any $\varepsilon>0$, and any $q \in Q^{*}, \pi^{*}$ is called $(p, \varepsilon, q)$-optimal if $p\left\{I\left(\pi^{*}, q\right) \geqq I(\pi, q)-\varepsilon\right\}=1$ for every $\pi . \quad \pi^{*}$ is called $(\varepsilon, q)$-optimal if it is $(p, \varepsilon, q)$-optimal for every $p \in P(S)$, or, equivalently if $I\left(\pi^{*}, q\right) \geqq I(\pi, q)-\varepsilon$ for all $\pi$, and is called $q$-optimal if it is $(\varepsilon, q)$-optimal for every $\varepsilon>0$, or, equivalently if $I\left(\pi^{*}, q\right) \geqq I(\pi, q)$ for all $\tau$. 


\section{$\S 4$. Optimality.}

Lemma 4.1. For any $p \equiv P(S), \varepsilon>0$, and $q \equiv Q^{*}$, there is a $(p, \varepsilon, q)$-optimal Markov strategy.

The proof of this lemma is straightforward by replacing $q$ in Theorem 2 of [2] by $q_{i}$.

With any measurable $f_{n}$ from $S$ to $A$ and any $q_{j} \leqq Q(S \mid S A)$ we associate the operator $T_{n j}$ from $M(S)$ to $M(S)$ defined by

$$
T_{n j} u(s)=\int\left[r\left(s, f_{n}(s), t\right) \div \beta u(t)\right] d q_{j}\left(t \mid s, f_{n}(s)\right) .
$$

We shall call $T_{n j}$ the operator associated with $\left(f_{n}, q_{j}\right)$. With any Markov strategy $\pi=\left\{f_{1}, f_{2}, \cdots\right\}$ and any $q_{j} \in Q(S \mid S A)$ we associate the operator $U_{j}$ from $M(S)$ to $M(S)$ defined by

$$
U_{j} u(s)=\sup _{n} T_{n j} u(s),
$$

where $T_{n j}$ is the operator associated with $\left(f_{n}, q_{j}\right)$. We shall call $U_{j}$ the operator associated with $\left(\pi, q_{j}\right)$. Let $\left(f_{n}, q_{j}\right) \sim T_{n j}$ mean that $T_{n j}$ is the operator associated with $\left(f_{n}, q_{j}\right)$, and let $\left(\pi, q_{j}\right) \sim U_{j}$ mean that $U_{j}$ associated with $\left(\pi, q_{j}\right)$. The following properties of $T_{n j}$ are immediate from the definition.

THEOREM 4.1. (a) $T_{n j}$ is monotone; i.e. $u \leqq v$ implies $T_{n j} u \leqq T_{n j} v$.

(b) $T_{n j}(u+c)=T_{n j} u+\beta c$ for any constant $c$.

(c) If $\pi=\left\{f_{1}, f_{2}, \cdots\right\}$ is a Markov strategy and $\left(f_{n}, q_{n}\right) \cdots T_{n n}$, then $T_{11} T_{22} \cdots T_{n n} v$ $=I_{n}(\pi, q, v)$ for each $n$.

(d) If $\pi=\left\{f_{1}, f_{2}, \cdots\right\}$ is a Markov strategy and $\left(f_{n}, q_{n}\right) \sim T_{n n}$, then $T_{n n} I\left({ }^{n} \pi,{ }^{n} q\right)$ $=I\left({ }^{n-1} \pi,{ }^{n-1} q\right)$ for each $n$.

For any Markov $\pi=\left\{f_{1}, f_{2}, \cdots\right\}$ we shall say that measurable $f$ mapping $S$ into $A$ is $\pi$-generated if there is a zartition of $S$ into Borel sets $S_{1}, S_{2}, \cdots$ such that $f=f_{n}$ on $S_{n}$ and we shall say that a Markov $\pi^{\prime}=\left\{g_{1}, g_{2}, \cdots\right\}$ is $\pi$-generated if each measurable $g_{n}$ is $\pi$-generated. Let $F(\pi)$ denote the class of all $\pi$-generated measurable functions, and let $G(\pi)$ the class of all $\pi$-generated Markov strategies.

THEOREM 4.2. (a) Let $\pi$ be any Markov strategy. Then for every $q_{j} \in Q(S \mid S A)$, $\hat{T}_{j} u \leqq U_{j} u$ for any $\hat{f} \in F(\pi)$ where $\left(\hat{f}, q_{j}\right) \sim \hat{T}_{j}$.

(b) For any Markov $\pi$, any $\varepsilon>0$ and any $q_{j} \in Q(S \mid S A)$, there exists $\hat{f}_{j} \in F(\pi)$ such that $\hat{T}_{j j} u \geqq U_{j} u-\varepsilon$ where $\left(\hat{f}_{j}, q_{j}\right) \sim \hat{T}_{j, j}$.

Proof. (a) Let $\pi$ be any Markov strategy. For any $\hat{f} \in F(\pi)$, let $\left(\hat{f}, q_{j}\right) \cdots \hat{T}_{j}$. By the definition of $F(\pi)$, there exists a partition of $S$ into Borel sets $S_{1}, S_{2}, \cdots$ such that $\hat{f}=f_{n}$ on $S_{n}$. Then for $s \in S_{n}, \hat{T}_{j} u(s)=T_{n j} u(s) \leqq U_{j} u(s)$. This holds for each $n$, which implies that $T_{j} u(s) \leqq U_{j} u(s)$ for all $s$.

(b) Let $\pi=\left\{f_{1}, f_{2}, \cdots\right\}$ be any Markov strategy, and let $\left(f_{n}, q_{j}\right) \cdots T_{n j}$. We let $S_{n j}=\left\{s \mid T_{m j} u<U_{j} u-\varepsilon\right.$ for $\left.m \leqq n-1, T_{n j} u \geqq U_{j} u-\varepsilon\right\}$, and define $\hat{f}_{j}$ by $\hat{f}_{j}=f_{n}$ on $S_{n j}$. Then for $s \in S_{n j}, \quad \hat{T}_{j j} u=T_{n j} u \geqq U_{j} u-\varepsilon$, where $\left(\hat{f}_{j}, q_{j}\right) \sim \hat{T}_{j j}$. So $\hat{T}_{j j} u \geqq U_{j} u-\varepsilon$ everywhere. Obviously $\hat{f}_{j} \in F(\pi)$. Hence the theorem is proved.

For any Markov $\pi$ and any $q_{j} \in Q(S \mid S A)$, let $\left(\pi, q_{j}\right) \sim U_{j}$. We shall call $u_{j-1}^{*}$ $\equiv \lim _{n \rightarrow \infty} U_{j} U_{j+1} \cdots U_{n} u$ the limit point associated with $\left(\pi,{ }^{j-1} q\right)$, where $u \in M(S)$. Let 
$\left(\pi,{ }^{j-1} q\right) \sim u_{j-1}^{*}$ mean that $u_{j-1}^{*}$ is the limit point associated with $\left(\pi,{ }^{j-1} q\right)$. In particular let $u^{*}=u_{0}^{*}$.

THEOREM 4.3. (a) For any Markov $\pi$ and any $q_{j} \in Q(S \mid S A)$, let $(\pi, q) \leadsto u^{*}$. Then $I(\hat{\therefore}, q) \leqq u *$ for every $\hat{\leqslant} \in G(\pi)$.

(b) For any Markov $\pi$, any $\varepsilon>0$ and any $q_{j} \in Q(S \mid S A)$, there is $a \hat{\kappa} \in G(\pi)$ such that $I(\hat{\pi}, q) \geqq u^{*}-\varepsilon$, where $(\pi, q) \sim u^{*}$.

(c) If for each $j \geqq 0$ there is a $\left(\varepsilon,{ }^{j} q\right)$-optimal strategy, there is a $(\varepsilon /(1-\beta)$, q)-optimal Markov strategy, where $\varepsilon \geqq 0$.

(d) Let $\left(f \equiv a, q_{j}\right) \sim T_{a j}$. Then if for avery $\varepsilon>0$ and for every $j \geqq 0$ there is $a$ $\left(\varepsilon,{ }^{i} q\right)$-optimal strategy, there is a Markov strategy $\hat{\approx}$ such that the limit point $\hat{u}_{j}^{*}$ associated with $\left(\hat{\pi},{ }^{j} q\right)$ is a Baire function for each $j$ and it satisfies the equations $\hat{u}_{j-1}^{*}$ $=\sup _{a \in A} T_{a j} \hat{u}_{j}^{*}$ for $j=1,2, \cdots$.

(e) For each $j \geqq 0 j^{j} \pi^{*}$ is $a^{j} q$-optimal if and only if the reward of $\pi^{*}$ satisfies the optimality equations; $I\left({ }^{j-1} \pi^{*},{ }^{j-1} q\right)=\sup _{a \in A} T_{a j} I\left({ }^{j} \pi^{*},{ }^{j} q\right)$ for $j=1,2, \cdots$.

Proof. (a) Let $\pi$ be any Markov strategy. Let $\hat{\kappa}=\left\{g_{1}, g_{2}, \cdots\right\} \in G(\pi)$, and let $\left(g_{j}, q_{j}\right) \sim \hat{T}_{j}$. By Theorem 4.1 (d) we have $\hat{T}_{n n} I\left({ }^{n} \hat{*},{ }^{n} q\right)=I\left({ }^{n-1} \hat{\hat{*}},{ }^{n-1} q\right)$ for each $n$, and backward inductively $\hat{T}_{11} \hat{T}_{22} \cdots \hat{T}_{n n} I\left({ }^{n} \hat{\mathrm{A}},{ }^{n} q\right)=I(\hat{\mathrm{A}}, q)$, i. e. $\hat{T}_{11} \hat{T}_{22} \cdots \hat{T}_{n n} u_{n}=I(\hat{\mathrm{A}}, q)$, where $u_{n}=I\left({ }^{n} \hat{\pi},{ }^{n} q\right)$.

Since for any $u \in M(S)$

$$
\left\|\hat{T}_{11} \hat{T}_{22} \cdots \hat{T}_{n n} u_{n}-\hat{T}_{11} \hat{T}_{22} \cdots \hat{T}_{n n} u\right\| \leqq \beta^{n}\left\|u_{n}-u\right\| \leqq \beta^{n}(\|r\| /(1-\beta)+\|u\|),
$$

we have

$$
\left\|I(\hat{\kappa}, q)-\hat{T}_{11} \hat{T}_{22} \cdots \hat{T}_{n n} u\right\| \leqq \beta^{n}(\|r\| /(1-\beta) \div\|u\|) .
$$

Therefore $\hat{T}_{11} \hat{T}_{22} \cdots \hat{T}_{n n} u \rightarrow I(\hat{\kappa}, q)$ as $n \rightarrow \infty$. In virtue of Theorem 4.2 (a) $\hat{T}_{j j} u \leqq U_{j} u$ for each $j$, so that backward inductively $\hat{T}_{11} \hat{T}_{22} \cdots \hat{T}_{j j} u \leqq U_{1} U_{2} \cdots U_{j} u$ for each $j$, which implies that $I(\hat{\pi}, q) \leqq u^{*}$ letting $j \rightarrow \infty$.

(b) Let $\varepsilon^{\prime}=\varepsilon(1-\beta)$. Then, from Theorem 4.2 (b), there exists an $\hat{f}_{j} \in F(\pi)$ corresponding to $\left(q_{j}, u\right)$ for which $\hat{T}_{j j} u \geqq U_{j} u-\varepsilon^{\prime}$ where $\left(\hat{f}_{j}, q_{j}\right) \sim \hat{T}_{j j}$. Similary there is an $\hat{f}_{j-1} \in F(\pi)$ corresponding to $\left(q_{j-1}, U_{j} u-\varepsilon^{\prime}\right)$ for which $\hat{T}_{j-1, j-1}\left(U_{j} u-\varepsilon^{\prime}\right) \geqq U_{j-1}\left(U_{j} u-\varepsilon^{\prime}\right)$. Thus we verify inductively that

$$
\hat{T}_{11} \hat{T}_{22} \cdots \hat{T}_{j j} u \geqq U_{1} U_{2} \cdots U_{j} u-\varepsilon^{\prime}\left(1+\beta+\cdots+\beta^{j-1}\right) \quad \text { for all } j \geqq 1 .
$$

We conclude that $I(\hat{\bar{\kappa}}, q) \geqq u^{*}-\varepsilon^{\prime} /(1-\beta)=u^{*}-\varepsilon$, letting $j \rightarrow \infty$.

(c) Assume that $\pi^{* j}=\left\{\pi_{j 1}, \pi_{j 2}, \cdots\right\}$ is a $\left(\varepsilon,{ }^{j} q\right)$-optimal strategy for each $j \geqq 0$. From Lemma 2.1, for each $j$ there exists a degenerate $f_{j}$ such that

$$
\begin{aligned}
I\left(\pi^{* j-1},{ }^{j-1} q\right) & =\pi_{j-1},{ }_{1} q_{j}\left[r+\beta I\left({ }^{1} \pi^{* j-1},{ }^{j} q\right)\right] \leqq \pi_{j-1},{ }_{1} q_{j}\left[r+\beta\left\{I\left(\pi^{* j},{ }^{j} q\right)+\varepsilon\right\}\right] \\
& \leqq f_{j} q_{j}\left[r+\beta\left\{I\left(\pi^{* j},{ }^{j} q\right)+\varepsilon\right\}\right]=T_{j j} I\left(\pi^{* j},{ }^{j} q\right)+\beta \varepsilon
\end{aligned}
$$

where $\left(f_{j}, q_{j}\right) \sim T_{j j}$. We have inductively degenerate $f_{1}, f_{2}, \cdots, f_{j-1}$ for which

$$
T_{11} T_{22} \cdots T_{j j} I\left(\pi^{* j},{ }^{j} q\right) \geqq I\left(\pi^{* 0}, q\right)-\varepsilon\left(\beta+\beta^{2}+\cdots+\beta^{j}\right) \quad \text { for all } j \geqq 1 .
$$

Letting $j \rightarrow \infty$ yields $I(\hat{\pi}, q) \geqq I\left(\pi^{* 0}, q\right)-\beta \varepsilon /(1-\beta)$, where $\hat{\pi}=\left\{f_{1}, f_{2}, \cdots\right\}$.

Since $\pi^{* 0}$ is a $(\varepsilon, q)$-optimal strategy, $I\left(\pi^{* 0}, q\right) \geqq I(\pi, q)-\varepsilon$ for all $\pi$. Therefore $I(\hat{\pi}, q) \geqq I(\pi, q)-\varepsilon /(1-\beta)$ for all $\pi$, which implies that $\hat{\pi}$ is a $(\varepsilon /(1-\beta), q)$-optimal Markov 
strategy.

(d) From (c), the hypothesis implies that there is a $\left(1 / n,{ }^{i} q\right)$-optimal Markov strategy $\pi^{i n}$, say, for each $n, j$. Let $\hat{\approx}$ be a Markov strategy for which $\pi^{j n} \subseteq G(\hat{*})$ for all $j, n$ and let $\left(\hat{*},{ }^{i} q\right) \sim \hat{u}_{j}^{*}$.

From (a), we have $I\left(\pi,{ }^{j} q\right) \leqq \hat{u}_{j}^{*}$ for all $\pi \in G(\hat{\hat{*}})$. Since $\pi^{j n} \leqq G(\hat{\pi})$ for all $n, j$, $I\left(\pi^{j n},{ }^{j} q\right) \leqq \hat{u}_{j}^{*}$ for all $n, j$. But from the definition of $\pi^{j n}, I\left(\pi^{j n},{ }^{j} q\right) \geqq I\left(\pi,{ }^{j} q\right)-1 / n$ for all $\pi$. Therefore $\hat{\imath}_{j}^{*} \geqq I\left(\pi,{ }^{j} q\right)-1 / n$ for all $\pi$. Letting $n \rightarrow \infty$ yields $\hat{u}_{j}^{*} \geqq I\left(\pi,{ }^{j} q\right)$ for all $\pi$ and all $j$. Since from (b) there exists a $\pi^{n j} \in G(\pi)$ such that $I\left(\tilde{\pi}^{n j},{ }^{j} q\right) \geqq \hat{u}_{j}^{*}-1 / n$ for each $n, j$, we conclude that

$$
T_{a j} \hat{u}_{j}^{*} \leqq T_{a j}\left[I\left(\tilde{\pi}^{n j},{ }^{j} q\right)+1 / n\right]=I\left(\left(a, \tilde{\pi}^{n j}\right),{ }^{j-1} q\right)+\beta / n \leqq \hat{u}_{j-1}^{*}+\beta / n,
$$

which implies $\sup _{a \equiv A} T_{a j} \hat{u}_{j}^{*} \leqq \hat{u}_{j-1}^{*}$. On the other hand it holds that $\sup _{a=A} T_{a j} \hat{u}_{j}^{*} \geqq U_{j} \hat{u}_{j}^{*}$ $=\hat{u}_{j-1}^{*}$. Thus we have $\sup _{a \in A} T_{a j} \hat{u}_{j}^{*}=\hat{u}_{j-1}^{*}$ for each $j$.

(e) Assume that ${ }^{j} \pi^{*}$ is a ${ }^{j} q$-optimal strategy for each $j$. Then from (c) we may assume $\pi^{*}=\left\{f_{1}^{*}, f_{2}^{*}, \cdots\right\}$ is Markov without loss of generality. Hence $I\left({ }^{j-1} \pi^{*},{ }^{j-1} q\right)_{s_{0}}$ $=T_{f_{j}^{*}\left(s_{0}\right),}{ }_{j} I\left({ }^{j} \pi^{*},{ }^{j} q\right)_{s_{0}}$ for each $j$, so that $\left.I^{j-1} \pi^{*},{ }^{j-1} q\right)_{s_{0}} \leqq \sup _{a \in A} T_{a j} I\left({ }^{j} \pi^{*},{ }^{j} q\right)_{s_{0}}$ for each $j$. Since this holds for any $s_{0} \in S$, it follows that $I\left({ }^{j-1} \pi^{*},{ }^{j-1} q\right) \leqq \sup _{a=A} T_{a j} I\left({ }^{j} \pi^{*},{ }^{j} q\right)$ everywhere for each $j$. But $I\left({ }^{j-1} \pi^{*},{ }^{j-1} q\right) \geqq I\left(\left(a,{ }^{j} \pi^{*}\right),{ }^{j-1} q\right)=T_{a j} I\left({ }^{j} \pi^{*},{ }^{j} q\right)$ for every $j$ and for all $a \in A$, which yields that $I\left({ }^{j-1} \pi^{*},{ }^{j-1} q\right) \geqq \sup _{a \in A} T_{a j} I\left({ }^{j} \pi^{*},{ }^{j} q\right)$ for every $j$. Finally we obtain optimality equations for $\pi^{*} ; I\left({ }^{j-1} \pi^{*},{ }^{j-1} q\right)=\sup _{a: * A} T_{a j} I\left({ }^{j} \pi^{*},{ }^{j} q\right)$ for every $j$.

Conversely assume that $\pi^{*}$ satisfies the optimality equations. In virtue of Lemma 4.1, for any $p \in P(S), \varepsilon>0, q \in Q^{*}$, there is a $(p, \varepsilon, q)$-optimal Markov strategy $\hat{\pi}$ $=\left(\hat{f}_{1}, \hat{f}_{2}, \cdots\right)$, say, i. e. $p\{I(\hat{\pi}, q) \geqq I(\pi, q)-\varepsilon\}=1$ for all $\pi$. So in particular it holds that $I(\hat{\pi}, q)_{s_{0}} \geqq I(\pi, q)_{s_{0}}-\varepsilon$ for all $\pi$. Let $\left(\hat{f}_{j}, q_{j}\right) \sim \leadsto \hat{T}_{j j}$, then from the assumption $\hat{T}_{j j} I\left({ }^{j} \pi^{*},{ }^{j} q\right)$ $\leqq I\left({ }^{j-1} \pi^{*},{ }^{j-1} q\right)$ for every $j$, and backward inductively $\hat{T}_{11} \hat{T}_{22} \cdots \hat{T}_{j j} I\left({ }^{j} \pi^{*},{ }^{j} q\right) \leqq I\left(\pi^{*}, q\right)$ for every $j$. Letting $j \rightarrow \infty$ yields $I(\hat{\pi}, q) \leqq I\left(\pi^{*}, q\right)$.

Therefore we have

$$
I(\pi, q)_{s_{0}} \leqq I(\hat{\pi}, q)_{s_{0}}+\varepsilon \leqq I\left(\pi^{*}, q\right)_{s_{0}}+\varepsilon \quad \text { for all } \pi,
$$

and we have $I(\pi, q)_{s_{0}} \leqq I\left(\pi^{*}, q\right)_{s_{0}}$ for all $\pi$ by letting $\varepsilon \rightarrow 0$. This hold for any $s_{0} \in S$, hence $I(\pi, q) \leqq I\left(\pi^{*}, q\right)$ for all $\pi$, which implies $\pi^{*}$ is a $q$-optimal strategy. Similary we get ${ }^{j} \pi^{*}$ is a ${ }^{j} q$-optimal strategy for each $j$. This completes the proof.

The following Corollary is an immediate consequence of Theorem 4.3 (c).

COROLlARY. If there is $a^{j} q$-optimal strategy for each $j \geqq 0$, then there is a q-optimal Markov strategy.

We shall say that actions $a$ and $b$ are equivalent at $\left(s, q_{j}\right)$ if $r(s, a, \cdot)=r(s, b, \cdot)$ and $q_{j}(\cdot \mid s, a)=q_{j}(\cdot \mid s, a)$, i. e. if $T_{a j} u(s)=T_{b j} u(s)$ for all $u \in M(S)$. We shall say that actions $a$ and $b$ are equivalent at $(s, q)$ if $r(s, a, \cdot)=r(s, b, \cdot)$ and $q_{j}(\cdot \mid s, a)=q_{j}(\cdot \mid s, a)$ for all $q_{j}$ in $q$, i. e. if $T_{a j} u(s)=T_{b j} u(s)$ for all $u \in M(S)$ and for all $q_{j}$ in $q$. For any Markov $\pi=\left\{f_{1}, f_{2}, \cdots\right\}$ A will be called essentially countable by $\pi$ if for every $(s, a)$ there is an $n$ for which $f_{n}(s)$ is equivalent to $a$ at $(s, q)$. A will be called essentially finite by $\pi$ if there is a partition of $S$ into Borel sets $S_{1}, S_{2}, \cdots$ such that for every $(s, a)$ with $s \in S_{n}$, at least one of the actions $f_{1}(s), f_{2}(s), \cdots, f_{n}(s)$ is equivalent to $a$ at 
$(s, q)$.

LEMMA 4.2. If $A$ is essentially finite by $\pi=\left\{f_{1}, f_{2}, \cdots\right\}$, then for any $q_{j} \in Q(S \mid S-A)$ and any $u \in M(S)$ there exists $\hat{f}_{j} \in F(\pi)$ for which $\hat{T}_{j i} u=U_{j} u$ where $\left(\hat{f}_{j}, q_{j}\right) \leadsto \hat{T}_{j i}$ and $\left(\pi, q_{j}\right) \sim U_{j}$.

Proof. We let $S_{n j}=\left\{s \mid T_{m j} u<U_{j} u\right.$ for $\left.m \leqq n-1, T_{n j} u=U_{j} u\right\}$. Then $\left\{s_{n j}, n=1\right.$, $2, \cdots\}$ comes to be a partition of $S$, since in this case $U_{j} u=\max T_{n j} u$. We set $\hat{f}_{j}=f_{n}$ on $S_{n j}$. Then for $s \in S_{n j}, \hat{T}_{j j} u=T_{n j} u=U_{j} u$, which completes the proof.

THEOREM 4.4. (a) If $A$ is essentially countable by $\pi=\left\{f_{1}, f_{2}, \cdots\right\}$, there is a $(\varepsilon, q)$ optimal Markov strategy for every $\varepsilon>0$ and every $q \in Q^{*}$.

(b) If $A$ is essentially finite by $\pi$, there is a q-optimal Markov strategy for every $q \in Q^{*}$.

PROOF. (a) From the assumption, for any $u \in M(S) \sup _{n} T_{n j} u=U_{j} u=\sup _{a \in A} T_{a j} u$ for all $j$, where $\left(f_{n}, q_{j}\right) \sim T_{n j}$ and $\left(\pi, q_{j}\right) \sim U_{j}$. Thus $T_{a j} u \leqq U_{j} u$ for any $u \in M(S)$, all $a \boxminus A$ and all $j$.

On the other hand, from Lemma 4.1, for any $p \in P(S), \varepsilon>0$ and $q \in Q^{*}$ there is a $(p, \varepsilon, q)$-optimal Markov strategy, say $\hat{\pi}=\left\{\hat{f}_{1}, \hat{f}_{2}, \cdots\right\}$, which yields that

$$
I(\hat{\pi}, q)_{s_{0}} \geqq I(\pi, q)_{s_{0}}-\varepsilon \quad \text { for all } \pi .
$$

We let $\left(\hat{f}_{j}, q_{j}\right) \sim \hat{T}_{j j}$, then we have $\hat{T}_{11} \hat{T}_{22} \cdots \hat{T}_{j j} u \leqq U_{1} U_{2} \cdots U_{j} u$ for all $j$. Letting $j \rightarrow \infty$ yields $I(\hat{\wedge}, q) \leqq u^{*}$, where $(\pi, q) \sim u^{*}$.

Thus we have

$$
I(\pi, q)_{s_{0}} \leqq I(\hat{\pi}, q)_{s_{0}}+\varepsilon \leqq u^{*}\left(s_{0}\right)+\varepsilon \quad \text { for all } \pi .
$$

Letting $\varepsilon \rightarrow 0$ yields that $I(\pi, q)_{s_{0}} \leqq u^{*}\left(s_{0}\right)$ for all $\pi$. This holds for all $s_{0} \in S$, so that $I(\pi, q) \leqq u^{*}$ for all $\pi$.

In virtue of Theorem 4.3 (b), there is a $\tilde{\pi} \in G(\pi)$ for which $I(\tilde{\pi}, q) \geqq u^{*}-\varepsilon$. Therefore we have $I(\tilde{\pi}, q) \geqq I(\pi, q)-\varepsilon$ for all $\pi$. This $\tilde{\pi}$ is $(\varepsilon, q)$-optimal.

(b) From Lemma 4.2 , there is a Markov strategy $\hat{\pi}=\left\{\hat{f}_{1}, \hat{f}_{2}, \cdots\right\}$ with $\hat{f}_{j} \in F(\pi)$ for $j=1,2, \cdots$, such that

$$
\hat{T}_{11} \hat{T}_{22} \cdots \hat{T}_{j j} u=U_{1} U_{2} \cdots U_{j} u .
$$

Letting $j \rightarrow \infty$ yields $I(\hat{\pi}, q)=u^{*}$, where $(\pi, q) \sim u^{*}$.

But in the proof of (a) we see that $u^{*} \geqq I(\pi)$ for all $\pi$. Thus we have $I(\hat{\pi}, q)$ $\geqq I(\pi)$ for all $\pi$, which completes the proof.

\section{§. l-stationary strategy.}

In this section we shall be concerned with the case when it is known to us that $q_{j}$ varies in a cyclic manner.

We shall say that $q$ is $l$-stationary if there exist $q_{j}^{\prime} \mathbf{s} \in Q(S \mid S A),(j=1,2, \cdots, l)$, such that $q=\{\bar{q}, \bar{q}, \cdots\}$ where $\bar{q}=\left\{q_{1}, q_{2}, \cdots, q_{l}\right\}$. An $l$-stationary $q$ is denoted by $\bar{q}^{(\infty)}$. We shall say that a Markov strategy $\pi$ is $l$-stationary if there exist degenerate $f_{j}$ s, $(j=1,2, \cdots, l)$, such that $\pi=\{\bar{f}, \bar{f}, \cdots\}$ where $\bar{f}=\left\{f_{1}, f_{2}, \cdots, f_{l}\right\}$. An $l$-stationary strategy is denoted by $\bar{f}(\infty)$.

For any Markov $\pi=\left\{f_{1}, f_{2}, \cdots\right\}$ we let $U_{j}$ the operator associated with $\left(\pi, q_{j}\right)$ for 
$j=1,2, \cdots, l$. We shall call $\bar{U}_{l} \equiv U_{1} U_{2} \cdots U_{l}$ the operator associated with $(\pi, \bar{q})$ where $\bar{q}=\left\{q_{1}, q_{2}, \cdots, q_{l}\right\} . \quad(\pi, \bar{q}) \sim \bar{U}_{l}$ means that $\bar{U}_{t}$ is the operator associated with $(\pi, \bar{q})$.

The following Lemma is immediate from the definition of $U_{j}$.

LEMMA 5.1. (a) $U_{j}$ is monotone for each $j$.

(b) For any constant $c, U_{j}(u+c)=U_{j} u+\beta c$.

THEOREM 5.1. $\bar{U}_{l}$, the operator associated with $(\pi, \bar{q})$, is a contraction with a contraction coefficient $\beta^{l}$, i.e. $\left\|\bar{U}_{l} u-\bar{U}_{l} v\right\| \leqq \beta^{l}\|u-v\|$, where $\|u\|=\sup _{s}|u(s)|$.

PROOF. From the difinition of a norm we have $v \leqq u+\|u-v\|$. In virtue of Lemma 5.1 it follows that

and so

$$
U_{l} u \leqq U_{\imath} u+\beta\|u-v\|,
$$

Similarly we have

$$
U_{l} v-U_{l} u \leqq \beta\|u-v\|
$$

$$
U_{l-1} U_{l} v-U_{l-1} U_{l} u \leqq \beta^{2}\|u-v\|,
$$

and inductively we have

$$
U_{1} U_{2} \cdots U_{l} v-U_{1} U_{2} \cdots U_{\imath} u \leqq \beta^{l}\|u-v\|,
$$

which shows that $\bar{U}_{l} v-\bar{U}_{\imath} u \leqq \beta^{l}\|u-v\|$. Interchange $u$ and $v$ to obtain $\bar{U}_{l} u-\bar{U}_{\iota} v$ $\leqq \beta^{l}\|u-v\|$. Thus we have $\left\|\bar{U}_{l} u-\bar{U}_{l} v\right\| \leqq \beta^{l}\|u-v\|$, completing the proof.

The general properties of optimal plans for $l$-stationary $q$ are contained in the following theorem.

THEOREM 5.2. (a) For any Markov $\pi$ and any l-stationary $q=\bar{q}^{(\infty)}$, let $(\pi, \bar{q}) \sim \bar{U}_{l}$. And let $\bar{u}_{l}^{*}$ be a fixed point of $\bar{U}_{l}$. Then $I\left(\hat{\pi}, \bar{q}^{(\infty)}\right) \leqq \bar{u}_{l}^{*}$ for every $\hat{\pi} \in G(\pi)$.

(b) For any Markov $\pi$ and any l-stationary $q=\bar{q}^{(\infty)}$, let $(\pi, \bar{q}) \sim \bar{U}_{l}$. And let $\bar{u}_{l}^{*}$ be a fixed point of $\bar{U}_{l}$. Then for every $\varepsilon>0$ there is a l-stationary strategy $\bar{f}^{(\infty)} \in G(\pi)$ for which $I\left(\bar{f}^{(\infty)}, \bar{q}^{(\infty)}\right) \geqq \bar{u}_{l}^{*}-\varepsilon$.

(c) For any $p \in P(S), \varepsilon>0$ and l-stationary $q=\bar{q}^{(\infty)}$, there is a $\left(p, \varepsilon, \bar{q}^{(\infty)}\right)$-optimal l-stationary strategy.

(d) For any $\varepsilon \geqq 0$, if there is a $\left(\varepsilon,\left(q_{j}, q_{j+1}, \cdots, q_{l}, \bar{q}^{(\infty)}\right)\right)$-optimal strategy for $j=1,2, \cdots, l$, then there is a $\left(\varepsilon /(1-\beta), \bar{q}^{(\infty)}\right)$-optimal l-stationary strategy, where $\bar{q}=\left\{q_{1}\right.$, $\left.q_{2}, \cdots, q_{l}\right\}$.

(e) Let $q$ be l-stationary. Then ${ }^{j} \pi^{*}$ is ${ }^{j} q$-optimal for $j=1,2, \cdots, l$ if and only if the expected reward of $\pi^{*}$ statisfies the optimallity equations; $I\left({ }^{j-1} \pi^{*},{ }^{j-1} q\right)=\sup _{a \in A} T_{a j} I^{(j} \pi^{*}$, ${ }^{j} q$ ) for $j=1,2, \cdots, l$.

Proof. (e) is immediate from (e) of Theorem 4.3. We shall prove (a) (d) only.

(a) Let $\hat{\pi}=\left\{q_{1}, q_{2}, \cdots\right\}$ be any $\pi$-generated strategy. Let $\left(g_{j}, q_{j}\right) \sim \hat{T}_{j j}$. Then, as stated in the proof of Theorem 4.3 (a), if holds that $\hat{T}_{11} \hat{T}_{22} \cdots \hat{T}_{j j} u \rightarrow I\left(\pi, \bar{q}^{(\infty)}\right)$ as $j \rightarrow \infty$ for all $u \in M(S)$.

Since $g_{i} \in F(\pi)$ for $i=1,2, \cdots$, from Theorem 4.2 (a), it follows that

$$
\hat{T}_{m l+1,1} \hat{T}_{m l+2,2} \cdots \hat{T}_{(m+1) l, l} u \leqq U_{1} U_{2} \cdots U_{l} u=\bar{U}_{l} u
$$

for each $m$ and for any $u \in M(S)$. Thus in particular

$$
\hat{T}_{m l+1,1} \hat{T}_{m l+2,2} \cdots \hat{T}_{(m+1) l, l} \bar{u}_{l}^{*} \leqq \vec{U}_{\imath} \bar{u}_{l}^{*}=\bar{u}_{l}^{*}
$$


for each $m$. Inductively we have

$$
\prod_{m=0}^{n}\left(\hat{T}_{m l+1,1} \hat{T}_{m l+2,2} \cdots \hat{T}_{(m+1) l, l}\right) \bar{u}_{l}^{*} \leqq \bar{u}_{l}^{*} .
$$

Letting $n \rightarrow \infty$ yields that $I\left(\hat{\cdots}, \bar{q}^{(\infty)}\right) \leqq \bar{u}_{i}^{*}$. This hold for all $\hat{\kappa} \in G(\pi)$.

(b) Let $\varepsilon^{\prime}=\varepsilon(1-\beta)$. In accordance with Theorem 4.2 (b) there is an $\hat{f}_{l} \in F(\pi)$ corresponding to $\left(q_{l}, \bar{u}_{l}^{*}\right)$ for which

$$
\hat{T}_{l \imath} \bar{u}_{l}^{*} \geqq U_{l} \bar{u}_{l}^{*}-\varepsilon^{\prime},
$$

where $\left(\hat{f}_{l}, q_{l}\right) \sim \hat{T}_{\iota l}$ and $\left(\pi, q_{l}\right) \sim U_{l}$. Similarly there is an $\hat{f}_{l-1} \in F(\pi)$ corresponding to $\left(q_{l-1}, U_{l} \bar{u}_{l}^{*}-\varepsilon^{\prime}\right)$ for which

$$
\hat{T}_{l-1, l-1}\left(U_{l} \bar{u}_{l}^{*}-\varepsilon^{\prime}\right) \geqq U_{l-1}\left(U_{l} \bar{u}_{l}^{*}-\varepsilon^{\prime}\right)-\varepsilon^{\prime},
$$

where $\left(\hat{f}_{l-1}, q_{l-1}\right) \leadsto \hat{T}_{l-1, l-1}$ and $\left(\pi, q_{l-1}\right) \leadsto U_{l-1}$. Thus we have

by Lemma 5.1 (b).

$$
\hat{T}_{l-1, l-1} \hat{T}_{l l} \bar{u}_{l}^{*} \geqq U_{l-1} U_{l} \bar{u}_{l}^{*}-\varepsilon^{\prime}(1+\beta)
$$

By backward induction we obtain

$$
\hat{T}_{11} \hat{T}_{22} \cdots \hat{T}_{l l} \bar{u}_{l}^{*} \geqq U_{1} U_{2} \cdots U_{l} \bar{u}_{l}^{*}-\varepsilon^{\prime}\left(1+\beta+\cdots+\beta^{l-1}\right)=\bar{u}_{l}^{*}-\varepsilon^{\prime}\left(1+\beta+\cdots+\beta^{l-1}\right) .
$$

Again inductively we have

$$
\left(\hat{T}_{11} \hat{T}_{22} \cdots \hat{T}_{l l}\right)^{n} \bar{u}_{l}^{*} \geqq \bar{u}_{l}^{*}-\varepsilon^{\prime}\left(1+\beta+\cdots+\beta^{n l-1}\right) \quad \text { for all } n \geqq 1,
$$

from which it follows that $I\left(\bar{f}^{(\infty)}, \bar{q}^{(\infty)}\right) \geqq \bar{u}_{l}^{*}-\varepsilon^{\prime} /(1-\beta)=\bar{u}_{l}^{*}-\varepsilon$. This $\bar{f}^{(\infty)}$ is obviously $l$-stationary and $\pi$-generated.

(c) In virtue of Lemma 4.1 there is a $\left(p, \varepsilon / 2, \bar{q}^{(\infty)}\right)$-optimal Markov $\pi=\left\{f_{1}, f_{2}, \cdots\right\}$ for any $p \in P(S), \varepsilon>0$ and any $l$-stationary $\bar{q}^{(\infty)}$. Let $(\pi, \bar{q}) \sim \bar{U}_{l}$ and let $\bar{u}_{\imath}^{*}$ be a fixed point of $\bar{U}_{l}$. Then from (b) there is a $\pi$-generated $l$-stationary $\bar{f}^{(\infty)}$ for which

From (a) it follows that

$$
I\left(\bar{f}^{(\infty)}, \bar{q}^{(\infty)}\right) \geqq \bar{u}_{l}^{*}-\varepsilon / 2 .
$$

Thus we have

$$
I\left(\pi, \bar{q}^{(\infty)}\right) \leqq \bar{u}_{l}^{*}
$$

$$
I\left(\bar{f}^{(\infty)}, \bar{q}^{(\infty)}\right) \geqq \bar{u}_{l}^{*}-\varepsilon / 2 \geqq I\left(\pi, \bar{q}^{(\infty)}\right)-\varepsilon / 2 .
$$

On the other hand, since $\pi$ is $\left(p, \varepsilon / 2, \bar{q}^{(\infty)}\right)$-optimal, we have

$$
P\left\{I\left(\pi, \bar{q}^{(\infty)}\right) \geqq I\left(\pi^{\prime}, \bar{q}^{(\infty)}\right)-\varepsilon / 2\right\}=1 \text { for all } \pi^{\prime} .
$$

Hence it follows that

$$
P\left\{I\left(\bar{f}^{(\infty)}, \bar{q}^{(\infty)}\right) \geqq I\left(\pi^{\prime}, \bar{q}^{(\infty)}\right)-\varepsilon\right\}=1 \quad \text { for all } \pi^{\prime} .
$$

This $\bar{f}^{(\infty)}$ is $\left(p, \varepsilon, \bar{q}^{(\infty)}\right)$-optimal l-stationary.

(d) Let $\tilde{q}_{j}=\left(q_{j}, q_{j+1}, \cdots, q_{l}\right)$. Assume that there is a $\left.\left(\varepsilon, \tilde{q}_{j}, \tilde{q}^{(\infty)}\right)\right)$-optimal strategy, say $\pi^{* j}=\left\{\pi_{j 1}, \pi_{j 2}, \cdots\right\}$, for each $j=1,2, \cdots, l$.

In accordance with Lemma 2.1 , for each $j=1,2, \cdots$, there exists a degenerate $f_{j}$ for which

$$
\begin{aligned}
I\left(\pi^{* j},\left(\tilde{q}_{j}, \bar{q}^{(\infty)}\right)\right) & =\pi_{j 1} q_{j}\left[r+\beta I\left({ }^{1} \pi^{* j},\left(\tilde{q}_{j+1}, \bar{q}^{(\infty)}\right)\right)\right] \\
& \leqq \pi_{j_{1}} q_{j}\left[r+\beta\left\{I\left(\pi^{* j+1},\left(\tilde{q}_{j+1}, \bar{q}^{(\infty)}\right)\right)+\varepsilon\right\}\right]
\end{aligned}
$$




$$
\begin{aligned}
& \leqq f_{j} q_{j}\left[r+\beta\left\{I\left(\pi^{* j+1},\left(\tilde{q}_{j+1}, \bar{q}^{(\infty)}\right)\right)+\varepsilon\right\}\right] \\
& =T_{j j} I\left(\pi^{* j+1},\left(\check{q}_{j+1}, \bar{q}^{(\infty)}\right)\right)+\beta \varepsilon,
\end{aligned}
$$

where $\left(f_{j}, q_{j}\right) \leadsto T_{j j}$. Since $\left(\tilde{q}_{l * 1}, \bar{q}^{(\infty)}\right)=\bar{q}^{(\infty)}$ and so $\pi^{* l+1}=\pi^{* 1}$, inductively we have

$$
T_{11} T_{22} \cdots T_{l l} I\left(\pi^{* 1}, \bar{q}^{(\infty)}\right) \geqq I\left(\pi^{* 1}, \bar{q}^{(\infty)}\right)-\varepsilon\left(\beta+\beta^{2}+\cdots+\beta^{l}\right) .
$$

Again inductively we have

$$
\left(T_{11} T_{22} \cdots T_{l l}\right)^{n} I\left(\pi^{* 1}, \bar{q}^{(\infty)}\right) \geqq I\left(\pi^{* 1}, \bar{q}^{(\infty)}\right)-\varepsilon\left(\beta+\beta^{2}+\cdots+\beta^{n t}\right) \quad \text { for all } n .
$$

Letting $n \rightarrow \infty$ yiels that

$$
I\left(\bar{f}^{(\infty)}, \vec{q}^{(\infty)}\right) \geqq I\left(\pi^{* 1}, \bar{q}^{(\infty)}\right)-\varepsilon \beta /(1-\beta),
$$

where $\bar{f}=\left\{f_{1}, f_{2}, \cdots, f_{l}\right\}$.

But, since $\pi^{* 1}$ is $\left(\varepsilon, \bar{q}^{(\infty)}\right)$-optimal, it holds that

Thus we obtain

$$
I\left(\pi, \bar{q}^{(\infty)}\right) \leqq I\left(\pi^{* 1}, \bar{q}^{(\infty)}\right)+\varepsilon \quad \text { for all } \pi .
$$

$$
I\left(\pi, \bar{q}^{(\infty)}\right) \leqq I\left(\bar{f}^{(\infty)}, \bar{q}^{(\infty)}\right)+\varepsilon /(1-\beta) \text { for all } \pi,
$$

which implies that $\bar{f}^{(\infty)}$ is a $\left(\varepsilon /(1-\beta), \bar{q}^{(\infty)}\right)$-optimal $l$-stationary strategy.

\section{§6. Strong optimality.}

In this section we shall define another optimality more strengthened than that of preceding sections, and we shall investigate an existence and some properties of an optimal strategy in this sence.

We let $v_{q}^{*}=\sup I(\pi, q), \pi^{*}$ will be called a strong $(p, \varepsilon, q)$-optimal strategy if $p\left\{I\left(\pi^{*}, q\right) \geqq v_{q}^{*}-\varepsilon\right\} \stackrel{\pi}{=} 1$.

The following two theorems are immediate respectively from Theorem 4.3 and from the proof of Theorem 8.1 in [3] by replacing $(q, q, \cdots)$ by $\left(q_{1}, q_{2}, \cdots\right)$.

THEOREM 6.1. For any $p \in P(S)$, any $q \in Q^{*}$ and any strategy $\pi$, there is a Markov strategy $\tilde{\pi}$ for which $p I(\tilde{\pi}, q) \geqq p I(\pi, q)$.

THEOREM 6.2. For any $p \in P(S), \varepsilon>0$ and any $q \in Q^{*}$ there is a strong $(p, \varepsilon, q)$ optimal strategy.

The following theorem is an alalogy of Theorem 6.2 in Strauch [3]. We shall prove it directly from (a) and (b) of our Theorem 4.3, whereas Strauch did by using the property of the "conservation".

THEOREM 6.3. For any sequence of Markov strategies $\left\{\pi^{j}, j=1,2, \cdots\right\}, \varepsilon>0$ and any $q \in Q^{*}$, there is a Markov $\hat{\pi}$ for which $I(\hat{\pi}, q) \geqq \sup _{j} I\left(\pi^{j}, q\right)-\varepsilon$.

PROOF. We can find a Markov strategy $\pi$ such that $\pi^{j} \in G(\pi)$ for $j=1,2, \cdots$. Let $(\pi, q) \sim u^{*}$ for this $\pi$.

Then, from (b) of Theorem 4.3, there is a Markov strategy $\hat{\approx} \in G(\pi)$ such that

$$
I(\hat{\pi}, q) \geqq u^{*}-\varepsilon .
$$

Since $\pi^{j} \in G(\pi)$ for $j=1,2, \cdots$, from (a) of Theorem 4.3, it holds that

which yield that

$$
u^{*} \geqq I\left(\pi^{j}, q\right) \quad \text { for } \quad j=1,2, \cdots,
$$




$$
u^{*} \geqq \sup _{j} I\left(\pi^{j}, q\right)
$$

Thus we have

$$
I(\hat{\kappa}, q) \geqq u^{*}-\varepsilon \geqq \sup _{j} I\left(\pi^{j}, q\right)-\varepsilon,
$$

which completes the proof.

Now let us show that a strong $(p, \varepsilon, q)$-optimal strategy exists, in fact, among the Markov strategies, by making use of the above theorems.

Theorem 6.4. For any $p \in P(S), \varepsilon>0$ and any $q \in Q^{*}$, there is a strong $(p, \varepsilon, q)$ optimal Markov strategy.

Proof. We let $\imath_{q}^{*}=\sup _{\pi} I(\pi, q)$. Then, in virtue of Theorem 6.2, there exists a strategy $\hat{\approx}$ (not necessarily Markov) for which $p\left\{I(\hat{*}, q) \geqq v_{q}^{*}-\varepsilon\right\}=1$. Hence we have

$$
p I(\hat{*}, q) \geqq p v_{q}^{*}-\varepsilon .
$$

From Theorem 6.1 there is a Markov strategy $\pi^{*}$ for this $\hat{\pi}$ such that

$$
p I\left(\pi^{*}, q\right) \geqq p I(\hat{\pi}, q) .
$$

Therefore it follows that there exists a Markov strategy $\pi^{*}$ for every $\varepsilon>0$ such that

$$
p I\left(\pi^{*}, q\right) \geqq p v_{q}^{*}-\varepsilon .
$$

Thus for each integer $m \geqq 1$ we can find a Markov strategy $\pi^{m}$ such that

$$
p I\left(\pi^{m}, q\right) \geqq p v_{q}^{*}-1 / m \text {. }
$$

Let $v_{q}^{\prime}=\sup _{m} I\left(\pi^{m}, q\right)$, then it folows that $p v_{q}^{\prime} \geqq p v_{q}^{*}-1 / m$. Letting $m \rightarrow \infty$ yields that $p v_{q}^{\prime} \geqq p v_{q}^{*}$. But $p v_{q}^{*} \geqq p v_{q}^{\prime}$, since $v_{q}^{*} \geqq v_{q}^{\prime}$ from the definitions of $v_{q}^{*}$ and $v_{q}^{\prime}$. Consequently $p v_{q}^{*}=p v_{q}^{\prime}$. Again from that $v_{q}^{*} \geqq v_{q}^{\prime}$, we have

$$
p\left\{v_{q}^{*}=v_{q}^{\prime}\right\}=1 .
$$

In virtue of Theorem 6.3 , for $\left\{\pi^{m}\right\}$ there is a Markov $\tilde{\pi}$ such that

Finally we have

$$
I(\tilde{\pi}, q) \geqq v_{q}^{\prime}-\varepsilon \text {. }
$$

$$
p\left\{I(\tilde{\pi}, q) \geqq v_{q}^{*}-\varepsilon\right\}=1,
$$

which say that this $\tilde{\pi}$ is a strong $(p, \varepsilon, q)$-optimal Markov strategy.

\section{$\S 7$. Improvements ef strategies.}

In this section we shall be concerned with several methods of strategy improvements.

THEOREM 7.1. If for a Markov strategy $\hat{\mathrm{\kappa}}=\left\{\hat{f}_{1}, \hat{f}_{2}, \cdots\right\}$

$$
I\left(\left(\hat{f}_{j},{ }^{i} \pi\right),{ }^{j-1} q\right) \geqq I\left({ }^{j-1} \pi,{ }^{j-1} q\right) \quad \text { for } \quad j=1,2, \cdots,
$$

then $I(\hat{\pi}, q) \geqq I(\pi, q)$.

Proof. We let $\left(\hat{f}_{j}, q_{j}\right) \cdots \hat{T}_{j j}$, then from the assumption we get

Consequently

$$
\left.\hat{T}_{j j} I\left({ }^{j} \pi,{ }^{j} q\right) \geqq I^{j-1} \pi,{ }^{j-1} q\right) \quad \text { for } \quad j=1,2, \cdots .
$$

$$
\hat{T}_{j-1, j-1} \hat{T}_{j j} I\left({ }^{j} \pi,{ }^{j} q\right) \geqq \hat{T}_{j-1, j-1} I\left({ }^{j-1} \pi,{ }^{j-1} q\right) \geqq I\left({ }^{j-2} \pi,{ }^{j-2} q\right) \quad \text { for } \quad j=1,2, \cdots,
$$


and inductively

$$
\hat{T}_{11} \hat{T}_{22} \cdots \hat{T}_{j j} I\left({ }^{i} \pi,{ }^{j} q\right) \geqq I\left({ }^{0} \pi,{ }^{0} q\right)=I(\pi, q) \quad \text { for } \quad j=1,2, \cdots .
$$

Letting $j \rightarrow \infty$ yields $I(\hat{\sim}, q) \geqq I(\pi, q)$.

THEOREM 7.2. If $I\left({ }^{n-1} \pi^{*},{ }^{n-1} q\right) \geqq I\left(\left(f,{ }^{n} \pi^{*}\right),{ }^{n-1} q\right)$ for all degenerate $f$ and for every $n$, then $\pi^{*}$ is q-optimal.

ProOf. Let $\pi=\left\{f_{1}, f_{2}, \cdots\right\}$ be any Markov strategy. By the assumption we have

$$
I\left({ }^{n-1} \pi^{*},{ }^{n-1} q\right) \geqq I\left(\left(f_{n}, \pi^{*}\right),{ }^{n-1} q\right) \quad \text { for } n=1,2, \cdots .
$$

Let $\left(\dot{f}_{j}, q_{j}\right) \sim T_{j j}$, then plainly

$$
\left.T_{11} T_{22} \cdots T_{n-1, n-1} I^{(n-1} \pi^{*},{ }^{n-1} q\right) \geqq T_{11} T_{22} \cdots T_{n-1, n-1} I\left(\left(f_{n},{ }^{n} \pi^{*}\right),{ }^{n-1} q\right) \quad \text { for } \quad n=1,2, \cdots,
$$

which implies that

$$
I\left(\left(f_{1}, f_{2}, \cdots, f_{n-1},{ }^{n-1} \pi^{*}\right), q\right) \geqq I\left(\left(f_{1}, f_{2}, \cdots, f_{n},{ }^{n} \pi^{*}\right), q\right) \quad \text { for } \quad n=1,2, \cdots .
$$

And inductively

$$
I\left(\pi^{*}, q\right) \geqq I\left(\left(f_{1}, f_{2}, \cdots, f_{n}, \pi^{*}\right), q\right) \quad \text { for } n=1,2, \cdots .
$$

Hence we have $I\left(\pi^{*}, q\right) \geqq I(\pi, q)$ by letting $n \rightarrow \infty$. This holds for any Markov strategy $\pi$. which completes the proof.

Now we shall define $G_{j}(s, \pi)$. Let $\left(f \equiv a, q_{j}\right) \sim T_{a j}$, then $G_{j}(s, \pi)$ is defined by

$$
G_{j}(s, \pi)=\left\{a \mid T_{a j} I\left({ }^{j} \pi,{ }^{j} q\right)>I\left({ }^{j-1} \pi,{ }^{j-1} q\right)\right\} \quad \text { for } j=1,2, \cdots, \text { and for } s \in S \text {. }
$$

We shall conclude with the following theorem.

THEOREM 7.3. (a) If for every $s \in S$ and for every $j G_{j}(s, \pi)$ is an emply set, then $\pi$ is a q-optimal strategy.

(b) Let $\pi=\left\{f_{1}, f_{2}, \cdots\right\}$ be any Markov strategy. If for each $j g_{j}\left(s_{0}\right) \in G_{j}\left(s_{0}, \pi\right)$ for some $s_{0}$ and $g_{j}(s)=f_{j}(s)$ for such that $g_{j}(s) \notin G_{j}(s, \pi)$, then $I(\hat{\pi}, q) \geqq I(\pi, q)$ where. $\hat{\pi}=\left\{q_{1}, q_{2}, \cdots\right\}$.

Proof. (a) From the assumption it follows that

$$
I\left({ }^{j-1} \pi,{ }^{j-1} q\right) \geqq I\left(\left(g_{j},{ }^{j} \pi\right),{ }^{j-1} q\right) \quad \text { for all } g_{j} \text { and every } j .
$$

Hence in virtue of Theorem $7.2 \pi$ is $q$-optimal.

(b) By the assumption, for each $j$ we have

and

$$
I\left(\left(g_{j},{ }^{j} \pi\right),{ }^{j-1} q\right)_{s_{0}}>I\left({ }^{j-1} \pi,{ }^{j-1} q\right)_{s_{0}}
$$

$$
I\left(\left(g_{j},{ }^{j} \pi\right),{ }^{j-1} q\right)_{s}=I\left({ }^{j-1} \pi,{ }^{j-1} q\right)_{s}
$$

for $s$ such that $g_{j}(s) \notin G_{j}(s, \pi)$, which implies that

$$
I\left(\left(g_{j},{ }^{j} \pi\right),{ }^{j-1} q\right) \geqq I\left({ }^{j-1} \pi,{ }^{j-1} q\right) \quad \text { for } \quad j=1,2, \cdots .
$$

Thus from Theorem $7.1 I(\hat{\pi}, q) \geqq I(\pi, q)$.

\section{References}

[1- R. Bellman, Dynamic Programming, Princeton Univ. Press, (1957).

[2] D. Blackwell, Discounted dynamic programming, Ann. Math. Statist., 36 (1965), $226-235$.

[3] R. E. Strauch, Negative dynamic programming, Ann. Math. Statist., 37 (1966), 871-890.

[4 M. Lot̀ve, Probability Theory, Van Nostrand, Princeton, (1960). 